\title{
Local Fractional Adomian Decomposition and Function Decomposition Methods for Laplace Equation within Local Fractional Operators
}

\author{
Sheng-Ping Yan, ${ }^{1}$ Hossein Jafari, ${ }^{2}$ and Hassan Kamil Jassim ${ }^{3}$ \\ ${ }^{1}$ School of Mechanics and Civil Engineering, China University of Mining and Technology, Xuzhou 221116, China \\ ${ }^{2}$ Department of Mathematical Sciences, University of South Africa, Pretoria, South Africa \\ ${ }^{3}$ Department of Mathematics, University of Mazandaran, Babolsar 47415-416, Iran \\ Correspondence should be addressed to Sheng-Ping Yan; spyan@cumt.edu.cn
}

Received 26 May 2014; Accepted 9 June 2014; Published 30 June 2014

Academic Editor: Xiao-Jun Yang

Copyright (c) 2014 Sheng-Ping Yan et al. This is an open access article distributed under the Creative Commons Attribution License, which permits unrestricted use, distribution, and reproduction in any medium, provided the original work is properly cited.

\begin{abstract}
We perform a comparison between the local fractional Adomian decomposition and local fractional function decomposition methods applied to the Laplace equation. The operators are taken in the local sense. The results illustrate the significant features of the two methods which are both very effective and straightforward for solving the differential equations with local fractional derivative.
\end{abstract}

\section{Introduction}

Many problems of physics and engineering are expressed by ordinary and partial differential equations, which are termed boundary value problems. We can mention, for example, the wave, the Laplace, the Klein-Gordon, the Schrodinger, the Advection, the Burgers, the Boussinesq, and the Fisher equations, and others [1].

Several analytical and numerical techniques were successfully applied to deal with differential equations, fractional differential equations, and local fractional differential equations [1-10]. The techniques include the heat-balance integral [11], the fractional Fourier [12], the fractional Laplace transform [12], the harmonic wavelet $[13,14]$, the local fractional Fourier and Laplace transform [15], local fractional variational iteration [16-18], the local fractional decomposition [19], and the generalized local fractional Fourier transform [20] methods.

In this paper, we investigate the application of local fractional Adomian decomposition method and local fractional function decomposition method for solving the local fractional Laplace equation $[21,22]$ with the different fractal conditions.
This paper is organized as follows. In Section 2, the basic mathematical tools are reviewed. Section 3 presents briefly the local fractional Adomian decomposition method and the local fractional function decomposition method. Section 4 presents solutions to the local fractional Laplace equation with differential fractal conditions.

\section{Mathematical Fundamentals}

We recall in this section the notations and some properties of the local fractional operators $[15-20,23,24]$.

Definition 1 (see $[15-20,23,24]$ ). The function $f(x)$ is local fractional continuous at $x=x_{0}$, if it is valid for

$$
\left|f(x)-f\left(x_{0}\right)\right|<\varepsilon^{\alpha}, \quad 0<\alpha \leq 1
$$

with $\left|x-x_{0}\right|<\delta$, for $\varepsilon>0$ and $\varepsilon \in R$. For $x \in(a, b)$, it is so called local fractional continuous on the interval $(a, b)$, denoted by $f(x) \in C_{\alpha}(a, b)$.

We notice that there are existence conditions of local fractional continuities that operating functions are right-hand 
and left-hand local fractional continuities. Meanwhile, the right-hand local fractional continuity is equal to its left-hand local fractional continuity. For more details, see [20].

Definition 2 (see $[15-20,23,24])$. The local fractional derivative of $f(x)$ at $x=x_{0}$ is defined as

$$
\begin{aligned}
D_{x}^{\alpha} f\left(x_{0}\right) & =\left.\frac{d^{\alpha}}{d x^{\alpha}} f(x)\right|_{x=x_{0}} \\
& =f^{(\alpha)}(x)=\lim _{x \rightarrow x_{0}} \frac{\Delta^{\alpha}\left(f(x)-f\left(x_{0}\right)\right)}{\left(x-x_{0}\right)^{\alpha}}
\end{aligned}
$$

where $\Delta^{\alpha}\left(f(x)-f\left(x_{0}\right)\right) \cong \Gamma(\alpha+1) \Delta\left(f(x)-f\left(x_{0}\right)\right)$. form

Local fractional derivative of high order is written in the

$$
f^{(k \alpha)}(x)=\overbrace{D_{x}^{\alpha} D_{x}^{\alpha} \cdots D_{x}^{\alpha}}^{k \text { times }} f(x) .
$$

And local fractional partial derivative of high order is written in the form

$$
\frac{\partial^{k \alpha} f(x, y)}{x^{k \alpha}}=\overbrace{\frac{\partial^{\alpha}}{\partial x^{\alpha}} \frac{\partial^{\alpha}}{\partial x^{\alpha}} \cdots \frac{\partial^{\alpha}}{\partial x^{\alpha}}}^{k \text { times }} f(x, y) .
$$

Definition 3 (see $[15-20,23,24]$ ). A partition of the interval $[a, b]$ is denoted by $\left(t_{j}, t_{j+1}\right), j=0, \ldots, N-1, t_{0}=a$ and $t_{N}=b$ with $\Delta t_{j}=t_{j+1}-t_{j}$ and $\Delta t=\max \left\{\Delta t_{0}, \Delta t_{1}, \ldots\right\}$. Local fractional integral of $f(x)$ in the interval $[a, b]$ is given by

$$
\begin{aligned}
a_{b}^{(\alpha)} f(x) & =\frac{1}{\Gamma(1+\alpha)} \int_{a}^{b} f(t)(d t)^{\alpha} \\
& =\frac{1}{\Gamma(1+\alpha)} \lim _{\Delta t \rightarrow 0} \sum_{j=0}^{N-1} f\left(t_{j}\right)\left(\Delta t_{j}\right)^{\alpha} .
\end{aligned}
$$

If the functions are local fractional continuous, then the local fractional derivatives and integrals exist. Some properties of local fractional derivative and integrals are given in $[20]$.

Definition 4. Let $f(x)$ be $2 l$-periodic. For $k \in Z$ and $f(x) \in$ $C_{\alpha}(a, b)$, the local fraction Fourier series of $f(x)$ is defined as (see $[15,25])$

$$
f(x)=\frac{a_{0}}{2}+\sum_{k=1}^{\infty}\left(a_{k} \cos _{\alpha} \frac{\pi^{\alpha}(k x)^{\alpha}}{l^{\alpha}}+b_{k} \sin _{\alpha} \frac{\pi^{\alpha}(k x)^{\alpha}}{l^{\alpha}}\right),
$$

where $a_{k}=\left(1 / l^{\alpha}\right) \int_{-1}^{1} f(x) \cos _{\alpha}\left(\pi^{\alpha}(k x)^{\alpha} / l^{\alpha}\right)(d x)^{\alpha}$

$$
b_{k}=\frac{1}{l^{\alpha}} \int_{-1}^{1} f(x) \sin _{\alpha} \frac{\pi^{\alpha}(k x)^{\alpha}}{l^{\alpha}}(d x)^{\alpha}
$$

Definition 5. Let $(1 / \Gamma(1+\alpha)) \int_{0}^{\infty}|f(x)|(d x)^{\alpha}<k<\infty$. The Yang-Laplace transforms of $f(x)$ are given by $[15,22]$

$$
L_{\alpha}\{f(x)\}=f_{s}^{L, \alpha}(s)=\frac{1}{\Gamma(1+\alpha)} \int_{0}^{\infty} E_{\alpha}\left(-s^{\alpha} x^{\alpha}\right) f(x)(d x)^{\alpha},
$$$$
0<\alpha \leq 1
$$

where the latter integral converges and $s^{\alpha} \in R^{\alpha}$.

Definition 6. The inverse formula of the Yang-Laplace transforms of $f(x)$ is given by $[15,22]$

$$
\begin{array}{r}
L_{\alpha}^{-1}\left\{f_{s}^{L, \alpha}(s)\right\} \\
=f(t)=\frac{1}{(2 \pi)^{\alpha}} \int_{\beta-i \omega}^{\beta+i \omega} E_{\alpha}\left(s^{\alpha} x^{\alpha}\right) f_{s}^{L, \alpha}(s)(d s)^{\alpha}, \\
\quad 0<\alpha \leq 1,
\end{array}
$$

where $s^{\alpha}=\beta^{\alpha}+i^{\alpha} \omega^{\alpha}$; fractal imaginary unit $i^{\alpha}$ and $\operatorname{Re}(s)=$ $\beta>0$.

\section{Analytical Methods}

In order to illustrate two analytical methods, we investigate the nonlinear local fractional equation of order $2 \alpha$ as follows:

$$
\begin{aligned}
& \frac{\partial^{2 \alpha} u(x, t)}{\partial t^{2 \alpha}}+k_{1} \frac{\partial^{\alpha} u(x, t)}{\partial t^{\alpha}}+k_{2} \frac{\partial^{2 \alpha} u(x, t)}{\partial x^{2 \alpha}}+k_{3} \frac{\partial^{\alpha} u(x, t)}{\partial x^{\alpha}} \\
& \quad=f(x, t)
\end{aligned}
$$

with constants $k_{1}, k_{2}, k_{3}, 0<\alpha \leq 1$ and with boundary and initial conditions

$$
\begin{gathered}
u(0, t)=u(l, t)=0 \\
u(x, 0)=\varphi(x) \\
\frac{\partial^{\alpha} u(x, 0)}{\partial t^{\alpha}}=\psi(x) .
\end{gathered}
$$

3.1. Local Fractional Adomian Decomposition Method. We rewrite (10) in the following form:

$$
\begin{aligned}
& L_{t t}^{(2 \alpha)} u(x, t)+k_{1} L_{t}^{(\alpha)} u(x, t)+k_{2} L_{x x}^{(2 \alpha)} u(x, t)+k_{3} L_{x}^{(\alpha)} u(x, t) \\
& \quad=f(x, t) .
\end{aligned}
$$

are local fractional Fourier coefficients. 
Applying the inverse operator $L_{t t}^{(-2 \alpha)}$ to both sides of (12) yields

$$
\begin{gathered}
L_{t t}^{(-2 \alpha)} L_{t t}^{(2 \alpha)} u(x, t) \\
=L_{t t}^{(-2 \alpha)}\left(-k_{1} L_{s}^{(\alpha)} u(x, s)-k_{2} L_{x x}^{(2 \alpha)} u(x, s)\right. \\
\left.-k_{3} L_{x}^{(\alpha)} u(x, s)+f(x, s)\right) \\
u(x, t)=r(x, t)+L_{t t}^{(-2 \alpha)}(f(x, s)) \\
+L_{t t}^{(-2 \alpha)}\left(-k_{1} L_{s}^{(\alpha)} u(x, s)-k_{2} L_{x x}^{(2 \alpha)} u(x, s)\right. \\
\left.-k_{3} L_{x}^{(\alpha)} u(x, s)\right),
\end{gathered}
$$

where the term $r(x, t)$ is to be determined from the fractal initial conditions.

Now, we decompose the unknown function $u(x, t)$ as a sum of components defined by the series:

$$
u(x, t)=\sum_{n=0}^{\infty} u_{n}(x, t) .
$$

The components $u_{n}(x, t)$ are obtained by the recursive formula:

$$
\begin{gathered}
u_{0}(x, t)=r(x, t)+L_{t t}^{(-2 \alpha)}(f(x, s)) \\
u_{n+1}(x, t)=L_{t t}^{(-2 \alpha)}\left(-k_{1} L_{s}^{(\alpha)} u_{n}(x, s)-k_{2} L_{x x}^{(2 \alpha)} u_{n}(x, s)\right. \\
\left.-k_{3} L_{x}^{(\alpha)} u_{n}(x, s)\right), \quad n \geq 0 .
\end{gathered}
$$

3.2. Local Fractional Function Decomposition Method. According to the decomposition of the local fractional function, with respect to the system $\left\{\sin _{\alpha} n^{\alpha}(\pi x / l)^{\alpha}\right\}$, the following functions coefficients can be given by

$$
\begin{aligned}
u(x, t) & =\sum_{n=1}^{\infty} v_{n}(t) \sin _{\alpha} n^{\alpha}\left(\frac{\pi x}{l}\right)^{\alpha} \\
f(x, t) & =\sum_{n=1}^{\infty} f_{n}(t) \sin _{\alpha} n^{\alpha}\left(\frac{\pi x}{l}\right)^{\alpha} \\
\varphi(x) & =\sum_{n=1}^{\infty} C_{n} \sin _{\alpha} n^{\alpha}\left(\frac{\pi x}{l}\right)^{\alpha} \\
\psi(x) & =\sum_{n=1}^{\infty} D_{n} \sin _{\alpha} n^{\alpha}\left(\frac{\pi x}{l}\right)^{\alpha},
\end{aligned}
$$

where

$$
\begin{aligned}
f_{n}(t) & =\frac{2}{l^{\alpha}} \int_{0}^{1} f(x, t) \sin _{\alpha} n^{\alpha}\left(\frac{\pi x}{l}\right)^{\alpha}(d x)^{\alpha} \\
C_{n} & =\frac{2}{l^{\alpha}} \int_{0}^{1} \varphi(x) \sin _{\alpha} n^{\alpha}\left(\frac{\pi x}{l}\right)^{\alpha}(d x)^{\alpha} \\
D_{n} & =\frac{2}{l^{\alpha}} \int_{0}^{1} \varphi(x) \sin _{\alpha} n^{\alpha}\left(\frac{\pi x}{l}\right)^{\alpha}(d x)^{\alpha} .
\end{aligned}
$$

Substituting (16) into (10) implies that

$$
\begin{gathered}
\frac{\partial^{2 \alpha} v_{n}(t)}{\partial t^{2 \alpha}}+k_{1} \frac{\partial^{\alpha} v_{n}(t)}{\partial t^{\alpha}}+k_{2}\left(\frac{n \pi}{l}\right)^{2 \alpha} v_{n}(t)+k_{3}\left(\frac{n \pi}{l}\right)^{\alpha} v_{n}(t) \\
=f_{n}(t) \\
v_{n}(0)=C_{n}, \quad v_{n}^{\prime}(0)=D_{n} .
\end{gathered}
$$

Suppose that the Yang-Laplace transforms of functions $v_{n}(t)$ and $f_{n}(t)$ are $V_{n}(s)$ and $F_{n}(s)$, respectively. Then, we obtain

$$
\begin{aligned}
& s^{2 \alpha} V_{n}(s)-C_{n} s^{\alpha}-D_{n}+k_{1}\left(s^{\alpha} V_{n}(s)-C_{n}\right)+k_{2}\left(\frac{n \pi}{l}\right)^{2 \alpha} V_{n}(s) \\
& \quad+k_{3}\left(\frac{n \pi}{l}\right)^{\alpha} V_{n}(s)=F_{n}(s) .
\end{aligned}
$$

That is,

$$
\begin{aligned}
V_{n}(s)= & \frac{D_{n}+k_{1} C_{n}+C_{n} s^{\alpha}}{s^{2 \alpha}+k_{1} s^{\alpha}+k_{2}(n \pi / l)^{2 \alpha}+k_{3}(n \pi / l)^{\alpha}} \\
& +\frac{F_{n}(s)}{s^{2 \alpha}+k_{1} s^{\alpha}+k_{2}(n \pi / l)^{2 \alpha}+k_{3}(n \pi / l)^{\alpha}} .
\end{aligned}
$$

Hence, we have

$$
\begin{aligned}
& v_{n}(t) \\
& =L_{\alpha}^{-1}\left\{V_{n}(s)\right\} \\
& =\frac{1}{(2 \pi)^{\alpha}} \int_{\beta-i \omega}^{\beta+i \omega} E_{\alpha}\left(s^{\alpha} x^{\alpha}\right) V_{n}(s)(d s)^{\alpha} \\
& =\frac{1}{(2 \pi)^{\alpha}} \int_{\beta-i \omega}^{\beta+i \omega} E_{\alpha}\left(s^{\alpha} x^{\alpha}\right) \\
& \quad \times \frac{F_{n}(s)}{s^{2 \alpha}+k_{1} s^{\alpha}+k_{2}(n \pi / l)^{2 \alpha}+k_{3}(n \pi / l)^{\alpha}}(d s)^{\alpha} \\
& +\frac{1}{(2 \pi)^{\alpha}} \int_{\beta-i \omega}^{\beta+i \omega} E_{\alpha}\left(s^{\alpha} x^{\alpha}\right) \\
& \quad \times \frac{D_{n}+k_{1} C_{n}+C_{n} s^{\alpha}}{s^{2 \alpha}+k_{1} s^{\alpha}+k_{2}(n \pi / l)^{2 \alpha}+k_{3}(n \pi / l)^{\alpha}}(d s)^{\alpha} .
\end{aligned}
$$




$$
\begin{aligned}
& \text { Let } v_{n}(t)=v_{1, n}(t)+v_{2, n}(t) \\
& v_{1, n}(t) \\
& =\frac{1}{(2 \pi)^{\alpha}} \int_{\beta-i \omega}^{\beta+i \omega} E_{\alpha}\left(s^{\alpha} x^{\alpha}\right) \\
& \times \frac{F_{n}(s)}{s^{2 \alpha}+k_{1} s^{\alpha}+k_{2}(n \pi / l)^{2 \alpha}+k_{3}(n \pi / l)^{\alpha}}(d s)^{\alpha} \\
& v_{2, n}(t) \\
& =\frac{1}{(2 \pi)^{\alpha}} \int_{\beta-i \omega}^{\beta+i \omega} E_{\alpha}\left(s^{\alpha} x^{\alpha}\right) \\
& \times \frac{D_{n}+k_{1} C_{n}+C_{n} s^{\alpha}}{s^{2 \alpha}+k_{1} s^{\alpha}+k_{2}(n \pi / l)^{2 \alpha}+k_{3}(n \pi / l)^{\alpha}}(d s)^{\alpha} .
\end{aligned}
$$

Hence, we get

$$
\begin{aligned}
& V_{1, n}(s)=\frac{F_{n}(s)}{s^{2 \alpha}+k_{1} s^{\alpha}+k_{2}(n \pi / l)^{2 \alpha}+k_{3}(n \pi / l)^{\alpha}} \\
& V_{2, n}(s)=\frac{D_{n}+k_{1} C_{n}+C_{n} s^{\alpha}}{s^{2 \alpha}+k_{1} s^{\alpha}+k_{2}(n \pi / l)^{2 \alpha}+k_{3}(n \pi / l)^{\alpha}} .
\end{aligned}
$$

Then, making use of (8) and (9) and rearranging integration sequence, we have the following several formulas about $v_{1, n}(t)$ and $v_{2, n}(t)$.

$$
\begin{aligned}
& \text { If }-(1 / 4) k_{1}^{2}+k_{2}(n \pi / l)^{2 \alpha}+k_{3}(n \pi / l)^{\alpha}>0 \text {, then } \\
& s^{2 \alpha}+k_{1} s+k_{2}\left(\frac{n \pi}{l}\right)^{2 \alpha}+k_{3}\left(\frac{n \pi}{l}\right)^{\alpha}=\left(s^{\alpha}+\frac{k_{1}}{2}\right)^{2}+D_{n}^{\prime},
\end{aligned}
$$

where $D_{n}^{\prime}=\sqrt{-(1 / 4) k_{1}^{2}+k_{2}(n \pi / l)^{2 \alpha}+k_{3}(n \pi / l)^{\alpha}}$.

Then, we get

$$
\begin{aligned}
v_{1, n}(t)= & \frac{1}{\Gamma(1+\alpha) D_{n}^{\prime}} \\
& \times \int_{0}^{t} E_{\alpha}\left(\frac{-k_{1} \tau^{\alpha}}{2^{\alpha}}\right) \sin _{\alpha}\left(D_{n}^{\prime} \tau^{\alpha}\right) f_{n}(t-\tau)(d \tau)^{\alpha} \\
v_{2, n}(t)= & C_{n} E_{\alpha}\left(\frac{-k_{1} t^{\alpha}}{2^{\alpha}}\right) \cos _{\alpha}\left(D_{n}^{\prime} t^{\alpha}\right) \\
& +\left(D_{n}+k_{1} C_{n}-\frac{k_{1}}{2}\right) E_{\alpha}\left(\frac{-k_{1} t^{\alpha}}{2^{\alpha}}\right) \sin _{\alpha}\left(D_{n}^{\prime} t^{\alpha}\right) .
\end{aligned}
$$

In case $-(1 / 4) k_{1}^{2}+k_{2}(n \pi / l)^{2 \alpha}+k_{3}(n \pi / l)^{\alpha}<0$ and $-(1 / 4) k_{1}^{2}+$ $k_{2}(n \pi / l)^{2 \alpha}+k_{3}(n \pi / l)^{\alpha}=0$, see [26].

\section{Solutions of Local Fractional Laplace Equation in Fractal Time-Space}

In this section, two examples for Laplace equation are presented in order to demonstrate the simplicity and the efficiency of the above methods.
The local fractional Laplace equation (see [21]) is one of the important differential equations with local fractional derivatives. In the following, we consider solutions to local fractional Laplace equations in fractal time-space.

Example 7. Consider the following local fractional Laplace equation:

$$
\frac{\partial^{2 \alpha} u(x, t)}{\partial t^{2 \alpha}}+\frac{\partial^{2} u(x, t)}{\partial x^{2 \alpha}}=0
$$

subject to the fractal value conditions

$$
u(x, 0)=-E_{\alpha}\left(x^{\alpha}\right), \quad \frac{\partial^{\alpha} u(x, 0)}{\partial t^{\alpha}}=0
$$

According to formula (15), we have

$$
\begin{gathered}
u_{0}(x, t)=r(x, t)+L_{t t}^{(-2 \alpha)}(f(x, s)), \\
u_{n+1}(x, t)=L_{t t}^{(-2 \alpha)}\left(-k_{1} L_{s}^{(\alpha)} u_{n}(x, s)-k_{2} L_{x x}^{(2 \alpha)} u_{n}(x, s)\right. \\
\left.-k_{3} L_{x}^{(\alpha)} u_{n}(x, s)\right),
\end{gathered}
$$

where

$$
u_{0}(x, t)=-E_{\alpha}\left(x^{\alpha}\right) .
$$

Hence, from (29) we obtain

$$
\begin{array}{r}
u_{n+1}(x, t)=L_{t t}^{(-2 \alpha)}\left\{-L_{x x}^{(2 \alpha)} u_{n}(x, s)\right\} \\
={ }_{0} I_{t}^{(\alpha)}{ }_{0} I_{t}^{(\alpha)}\left\{-\frac{\partial^{2 \alpha} u_{n}(x, s)}{\partial x^{2 \alpha}}\right\}, \\
n \geq 1,
\end{array}
$$

where

$$
u_{0}(x, t)=-E_{\alpha}\left(x^{\alpha}\right) .
$$

Making use of (31), we present

$$
\begin{aligned}
u_{1}(x, t) & ={ }_{0} I_{t}^{(\alpha)}{ }_{0} I_{t}^{(\alpha)}\left\{-\frac{\partial^{2 \alpha} u_{0}(x, s)}{\partial x^{2 \alpha}}\right\} \\
& ={ }_{0} I_{t}^{(\alpha)}{ }_{0} I_{t}^{(\alpha)}\left\{E_{\alpha}\left(x^{\alpha}\right)\right\} \\
& =\frac{t^{2 \alpha}}{\Gamma(1+2 \alpha)} E_{\alpha}\left(x^{\alpha}\right)
\end{aligned}
$$




$$
\begin{aligned}
u_{2}(x, t) & ={ }_{0} I_{t}^{(\alpha)}{ }_{0} I_{t}^{(\alpha)}\left\{-\frac{\partial^{2 \alpha} u_{1}(x, s)}{\partial x^{2 \alpha}}\right\} \\
& ={ }_{0} I_{t}^{(\alpha)}{ }_{0} I_{t}^{(\alpha)}\left\{-\frac{t^{2 \alpha}}{\Gamma(1+2 \alpha)} E_{\alpha}\left(x^{\alpha}\right)\right\} \\
& =-\frac{t^{4 \alpha}}{\Gamma(1+4 \alpha)} E_{\alpha}\left(x^{\alpha}\right) \\
u_{3}(x, t) & ={ }_{0} I_{t}^{(\alpha)}{ }_{0} I_{t}^{(\alpha)}\left\{-\frac{\partial^{2 \alpha} u_{2}(x, s)}{\partial x^{2 \alpha}}\right\} \\
& ={ }_{0} I_{t}^{(\alpha)}{ }_{0} I_{t}^{(\alpha)}\left\{\frac{t^{4 \alpha}}{\Gamma(1+4 \alpha)} E_{\alpha}\left(x^{\alpha}\right)\right\} \\
& =\frac{t^{6 \alpha}}{\Gamma(1+6 \alpha)} E_{\alpha}\left(x^{\alpha}\right) .
\end{aligned}
$$

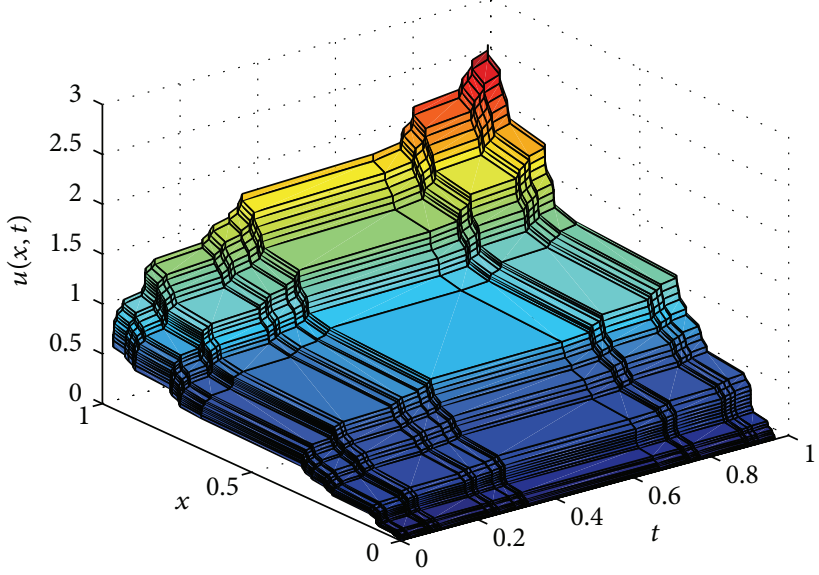

FIGURE 1: Exact solution for local fractional Laplace equation with fractal dimension $\alpha=\ln 2 / \ln 3$.

Proceeding in this manner, we get

$$
u_{n}(x, t)=E_{\alpha}\left(x^{\alpha}\right)(-1)^{n+1} \frac{t^{2 n \alpha}}{\Gamma(1+2 n \alpha)} .
$$

Thus, the final solution reads as follows:

$$
\begin{aligned}
u(x, t)= & \sum_{n=0}^{\infty} u_{n}(x, t)=u_{0}(x, t)+u_{1}(x, t)+u_{2}(x, t)+\cdots \\
= & E_{\alpha}\left(x^{\alpha}\right)\left[-1+\frac{t^{2 \alpha}}{\Gamma(1+2 \alpha)}-\frac{t^{4 \alpha}}{\Gamma(1+4 \alpha)}\right. \\
& \left.+\frac{t^{6 \alpha}}{\Gamma(1+6 \alpha)} \cdots\right] \\
= & -E_{\alpha}\left(x^{\alpha}\right)\left[1-\frac{t^{2 \alpha}}{\Gamma(1+2 \alpha)}+\frac{t^{4 \alpha}}{\Gamma(1+4 \alpha)}\right. \\
= & \left.-\frac{t^{6 \alpha}}{\Gamma(1+6 \alpha)} \cdots\right] \\
= & E_{\alpha}\left(x^{\alpha}\right) \cos _{\alpha}\left(t^{\alpha}\right)
\end{aligned}
$$

Now, we solve Example 7 by using the local fractional function decomposition method.

We suppose that

$$
\begin{gathered}
u(x, t)=\sum_{n=1}^{\infty} v_{n}(t) E_{\alpha}\left(n^{\alpha} x^{\alpha}\right) \\
f(x, t)=\sum_{n=1}^{\infty} f_{n}(t) E_{\alpha}\left(n^{\alpha} x^{\alpha}\right) \\
\varphi(x)=\sum_{n=1}^{\infty} C_{n} E_{\alpha}\left(n^{\alpha} x^{\alpha}\right) \\
\psi(x)=\sum_{n=1}^{\infty} D_{n} E_{\alpha}\left(n^{\alpha} x^{\alpha}\right)
\end{gathered}
$$

which leads to

$$
\begin{gathered}
f_{n}(t)=0, \quad \forall n ; \quad C_{n}=0, \quad n \neq 1 ; \quad C_{1}=-1 ; \\
D_{n}=0, \quad \forall n .
\end{gathered}
$$

Contrasting (28) with (36), we directly get $k_{1}=0, k_{2}=1$, and $k_{3}=0$ and

$$
\begin{aligned}
D_{n}^{\prime}=0, \quad n \neq 1 ; \quad D_{1}^{\prime}=1 & v_{n}(t)=0, \quad n \neq 1 \\
v_{1,1}(t)= & \frac{1}{\Gamma(1+\alpha) D_{1}^{\prime}} \\
\times & \int_{0}^{t} E_{\alpha}\left(\frac{-k_{1} \tau^{\alpha}}{2^{\alpha}}\right) \sin _{\alpha}\left(D_{1}^{\prime} \tau^{\alpha}\right) f_{1}(t-\tau)(d \tau)^{\alpha}=0 \\
v_{2,1}(t)= & C_{1} E_{\alpha}\left(\frac{-k_{1} t^{\alpha}}{2^{\alpha}}\right) \cos _{\alpha}\left(D_{1}^{\prime} t^{\alpha}\right) \\
& +\left(D_{1}+k_{1} C_{1}-\frac{k_{1}}{2}\right) E_{\alpha}\left(\frac{-k_{1} t^{\alpha}}{2^{\alpha}}\right) \sin _{\alpha}\left(D_{1}^{\prime} t^{\alpha}\right) \\
= & -\cos _{\alpha}\left(t^{\alpha}\right) .
\end{aligned}
$$

Conclusively, we get

$$
v_{1}(t)=v_{1,1}(t)+v_{2,1}(t)=-\cos _{\alpha}\left(t^{\alpha}\right) .
$$

Thus, we obtain

$$
u(x, t)=-E_{\alpha}\left(x^{\alpha}\right) \cos _{\alpha}\left(t^{\alpha}\right)
$$

and its graph is shown in Figure 1.

Example 8. We consider the following local fractional Laplace equation:

$$
\frac{\partial^{2 \alpha} u(x, t)}{\partial t^{2 \alpha}}+\frac{\partial^{2 \alpha} u(x, t)}{\partial x^{2 \alpha}}=0
$$


subject to the fractal value conditions

$$
u(x, 0)=0, \quad \frac{\partial^{\alpha} u(x, 0)}{\partial t^{\alpha}}=-E_{\alpha}\left(x^{\alpha}\right) .
$$

Now we can structure the same local fractional iteration procedure (15). Hence, we have

$$
\begin{aligned}
u_{0}(x, t) & =-\frac{t^{\alpha}}{\Gamma(1+\alpha)} E_{\alpha}\left(x^{\alpha}\right) \\
u_{1}(x, t) & ={ }_{0} I_{t}^{(\alpha)}{ }_{0} I_{t}^{(\alpha)}\left\{-\frac{\partial^{2 \alpha} u_{0}(x, s)}{\partial x^{2 \alpha}}\right\} \\
& ={ }_{0} I_{t}^{(\alpha)}{ }_{0} I_{t}^{(\alpha)}\left\{\frac{t^{\alpha}}{\Gamma(1+\alpha)} E_{\alpha}\left(x^{\alpha}\right)\right\} \\
& =\frac{t^{3 \alpha}}{\Gamma(1+3 \alpha)} E_{\alpha}\left(x^{\alpha}\right) \\
u_{2}(x, t) & ={ }_{0} I_{t}^{(\alpha)}{ }_{0} I_{t}^{(\alpha)}\left\{-\frac{\partial^{2 \alpha} u_{1}(x, s)}{\partial x^{2 \alpha}}\right\} \\
& ={ }_{0} I_{t}^{(\alpha)}{ }_{0} I_{t}^{(\alpha)}\left\{-\frac{t^{3 \alpha}}{\Gamma(1+3 \alpha)} E_{\alpha}\left(x^{\alpha}\right)\right\} \\
& =-\frac{t^{5 \alpha}}{\Gamma(1+5 \alpha)} E_{\alpha}\left(x^{\alpha}\right) \\
u_{3}(x, t) & ={ }_{0} I_{t}^{(\alpha)}{ }_{0} I_{t}^{(\alpha)}\left\{-\frac{\partial^{2 \alpha} u_{2}(x, s)}{\partial x^{2 \alpha}}\right\} \\
& ={ }_{0} I_{t}^{(\alpha)}{ }_{0} I_{t}^{(\alpha)}\left\{\frac{t^{5 \alpha}}{\Gamma(1+5 \alpha)} E_{\alpha}\left(x^{\alpha}\right)\right\} \\
& =\frac{t^{7 \alpha}}{\Gamma(1+7 \alpha)} E_{\alpha}\left(x^{\alpha}\right) .
\end{aligned}
$$

Finally, we can obtain the local fractional series solution as follows:

$$
u_{n}(x, t)=(-1)^{n+1} \frac{t^{(2 n+1) \alpha}}{\Gamma(1+(2 n+1) \alpha)} E_{\alpha}\left(x^{\alpha}\right) .
$$

Thus, the final solution reads as follows:

$$
\begin{aligned}
& u(x, t) \\
& =\sum_{n=0}^{\infty} u_{n}(x, t)=u_{0}(x, t)+u_{1}(x, t)+u_{2}(x, t)+\cdots \\
& =E_{\alpha}\left(x^{\alpha}\right)\left[-\frac{t^{\alpha}}{\Gamma(1+\alpha)}+\frac{t^{3 \alpha}}{\Gamma(1+3 \alpha)}-\frac{t^{5 \alpha}}{\Gamma(1+5 \alpha)} \cdots\right] \\
& =-E_{\alpha}\left(x^{\alpha}\right)\left[\frac{t^{\alpha}}{\Gamma(1+\alpha)}-\frac{t^{3 \alpha}}{\Gamma(1+3 \alpha)}+\frac{t^{5 \alpha}}{\Gamma(1+5 \alpha)} \cdots\right] \\
& =-E_{\alpha}\left(x^{\alpha}\right) \sin _{\alpha}\left(t^{\alpha}\right) .
\end{aligned}
$$

Now, we solve Example 8 by using the local fractional function decomposition method.

We suppose that

$$
\begin{gathered}
u(x, t)=\sum_{n=1}^{\infty} v_{n}(t) E_{\alpha}\left(n^{\alpha} x^{\alpha}\right) \\
f(x, t)=0=\sum_{n=1}^{\infty} f_{n}(t) E_{\alpha}\left(n^{\alpha} x^{\alpha}\right) \\
\varphi(x)=0=\sum_{n=1}^{\infty} C_{n} E_{\alpha}\left(n^{\alpha} x^{\alpha}\right) \\
\psi(x)=-E_{\alpha}\left(x^{\alpha}\right)=\sum_{n=1}^{\infty} D_{n} E_{\alpha}\left(n^{\alpha} x^{\alpha}\right)
\end{gathered}
$$

which leads to

$$
\begin{gathered}
f_{n}(t)=0, \quad \forall n ; \quad D_{n}=0, \quad n \neq 1 ; \quad D_{1}=-1 ; \\
C_{n}=0, \quad \forall n .
\end{gathered}
$$

Contrasting (28) with (36), we directly get $k_{1}=0, k_{2}=1$, and $k_{3}=0$ and

$$
\begin{gathered}
D_{n}^{\prime}=0, \quad n \neq 1 ; \quad D_{1}^{\prime}=1 \\
v_{n}(t)=0, \quad n \neq 1 \\
v_{1,1}(t)=\frac{1}{\Gamma(1+\alpha) D_{1}^{\prime}} \\
\times \int_{0}^{t} E_{\alpha}\left(\frac{-k_{1} \tau^{\alpha}}{2^{\alpha}}\right) \sin _{\alpha}\left(D_{1}^{\prime} \tau^{\alpha}\right) f_{1}(t-\tau)(d \tau)^{\alpha} \\
=0 \\
v_{2,1}(t)=C_{1} E_{\alpha}\left(\frac{-k_{1} t^{\alpha}}{2^{\alpha}}\right) \cos _{\alpha}\left(D_{1}^{\prime} t^{\alpha}\right) \\
+\left(D_{1}+k_{1} C_{1}-\frac{k_{1}}{2}\right) E_{\alpha}\left(\frac{-k_{1} t^{\alpha}}{2^{\alpha}}\right) \sin _{\alpha}\left(t^{\alpha}\right) \\
=-\sin _{\alpha}\left(t^{\alpha}\right) .
\end{gathered}
$$

Conclusively, we get

$$
v_{1}(t)=v_{1,1}(t)+v_{2,1}(t)=-\sin _{\alpha}\left(t^{\alpha}\right) .
$$

Thus, we obtain

$$
u(x, t)=-E_{\alpha}\left(x^{\alpha}\right) \sin _{\alpha}\left(t^{\alpha}\right)
$$

and its graph is given in Figure 2.

\section{Conclusions}

In this work solving the Laplace equations using the local fractional function decomposition method with local fractional operators is discussed in detail. Two examples of 


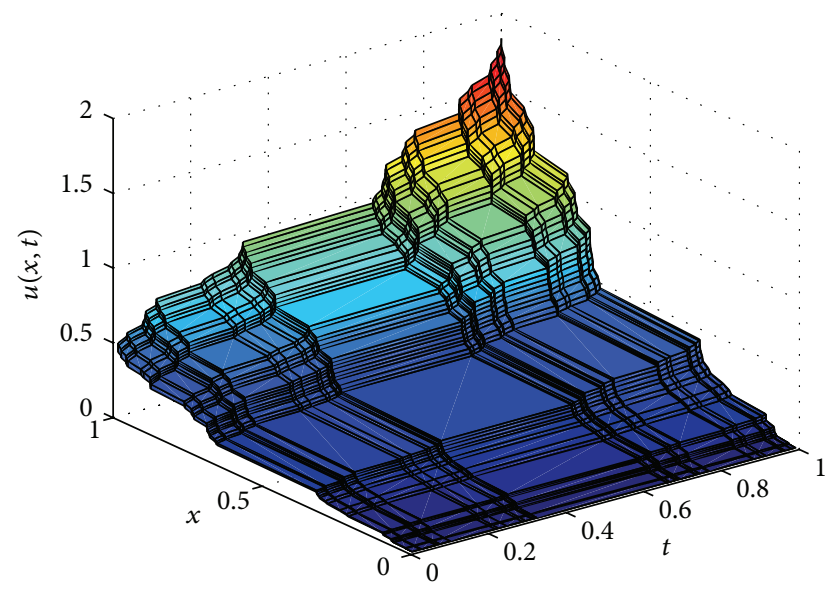

FIgURE 2: The plot of solution to local fractional Laplace equation with fractal dimension $\alpha=\ln 2 / \ln 3$.

applications of the local fractional Adomian decomposition method and local fractional function decomposition method to the local fractional Laplace equations are investigated in detail. The reliable obtained results are complementary with the ones presented in the literature.

\section{Conflict of Interests}

The authors declare that there is no conflict of interests regarding the publication of the paper.

\section{References}

[1] A. M. Wazwaz, Partial Differential Equations: Methods and Applications, Elsevier, Balkema, The Netherlands, 2002.

[2] W. R. Schneider and W. Wyss, "Fractional diffusion and wave equations," Journal of Mathematical Physics, vol. 30, no. 1, pp. 134-144, 1989.

[3] Z. Zhao and C. Li, "Fractional difference/finite element approximations for the time-space fractional telegraph equation," Applied Mathematics and Computation, vol. 219, no. 6, pp. 29752988, 2012.

[4] S. Momani, Z. Odibat, and A. Alawneh, "Variational iteration method for solving the space- and time-fractional KdV equation," Numerical Methods for Partial Differential Equations, vol. 24, no. 1, pp. 262-271, 2008.

[5] N. Laskin, "Fractional Schrödinger equation," Physical Review E: Statistical, Nonlinear, and Soft Matter Physics, vol. 66, no. 5, Article ID 056108, 2002.

[6] Y. Zhou and F. Jiao, "Nonlocal Cauchy problem for fractional evolution equations," Nonlinear Analysis. Real World Applications, vol. 11, no. 5, pp. 4465-4475, 2010.

[7] S. Momani and Z. Odibat, "Analytical solution of a timefractional Navier-Stokes equation by Adomian decomposition method," Applied Mathematics and Computation, vol. 177, no. 2, pp. 488-494, 2006.

[8] V. E. Tarasov, "Fractional heisenberg equation," Physics Letters A, vol. 372, no. 17, pp. 2984-2988, 2008.

[9] A. K. Golmankhaneh, A. K. Golmankhaneh, and D. Baleanu, "On nonlinear fractional KleinGordon equation," Signal Processing, vol. 91, no. 3, pp. 446-451, 2011.
[10] Z. Li, W. Zhu, and L. Huang, "Application of fractional variational iteration method to time-fractional Fisher equation," Advanced Science Letters, vol. 10, pp. 610-614, 2012.

[11] J. Hristov, "Heat-balance integral to fractional (half-time) heat diffusion sub-model," Thermal Science, vol. 14, no. 2, pp. 291-316, 2010.

[12] D. Baleanu, K. Diethelm, E. Scalas, and J. J. Trujillo, Fractional Calculus Models and Numerical Methods, vol. 3 of Series on Complexity, Nonlinearity and Chaos, World Scientific, Boston, Mass, USA, 2012.

[13] C. Cattani, "Harmonic wavelet solution of Poisson's problem," Balkan Journal of Geometry and Its Applications, vol. 13, no. 1, pp. 27-37, 2008.

[14] C. Cattani, "Harmonic wavelets towards the solution of nonlinear PDE," Computers \& Mathematics with Applications, vol. 50, no. 8-9, pp. 1191-1210, 2005.

[15] X. J. Yang, Local Fractional Functional Analysis and Its Applications, Asian Academic, Hong Kong, China, 2011.

[16] X. Yang and D. Baleanu, "Fractal heat conduction problem solved by local fractional variation iteration method," Thermal Science, vol. 17, no. 2, pp. 625-628, 2013.

[17] W.-H. Su, D. Baleanu, X.-J. Yang, and H. Jafari, "Damped wave equation and dissipative wave equation in fractal strings within the local fractional variational iteration method," Fixed Point Theory and Applications, vol. 2013, no. 89, 2013.

[18] Y. J. Yang, D. Baleanu, and X. J. Yang, "A local fractional variational iteration method for Laplace equation within local fractional operators," Abstract and Applied Analysis, vol. 2013, Article ID 202650, 6 pages, 2013.

[19] X. Yang, D. Baleanu, and W. Zhong, "Approximate solutions for diffusion equations on Cantor space-time," Proceedings of the Romanian Academy A, vol. 14, no. 2, pp. 127-133, 2013.

[20] X. J. Yang, Advanced Local Fractional Calculus and Its Applications, World Science, New York, NY, USA, 2012.

[21] A. Liangprom and K. Nonlaopon, "On the convolution equation related to the diamond Klein-Gordon operator," Abstract and Applied Analysis, vol. 2011, Article ID 908491, 16 pages, 2011.

[22] C. F. Liu, S. S. Kong, and S. J. Yuan, "Reconstructive schemes for variational iteration method within Yang-Laplace transform with application to fractal heat conduction problem," Thermal Science, vol. 17, no. 3, pp. 715-721, 2013.

[23] X. J. Yang, D. Baleanu, and J. A. T. Machado, "Mathematical aspects of Heisenberg uncertainty principle within local fractional Fourier analysis," Boundary Value Problems, no. 1, pp. 131146, 2013

[24] A. Yang, X. Yang, and Z. Li, "Local fractional series expansion method for solving wave and diffusion equations on Cantor sets," Abstract and Applied Analysis, vol. 2013, Article ID 351057, 5 pages, 2013.

[25] M. Hu, R. P. Agarwal, and X.-J. Yang, "Local fractional Fourier series with application to wave equation in fractal vibrating string," Abstract and Applied Analysis, vol. 2012, Article ID 567401, 15 pages, 2012.

[26] S. Q. Wang, Y. J. Yang, and H. K. Jassim, "Local fractional function decomposition method for solving inhomogeneous wave equations with local fractional derivative," Abstract and Applied Analysis, vol. 2014, Article ID 176395, 7 pages, 2014. 


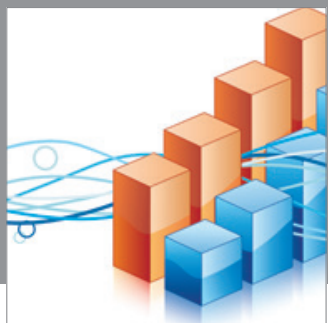

Advances in

Operations Research

mansans

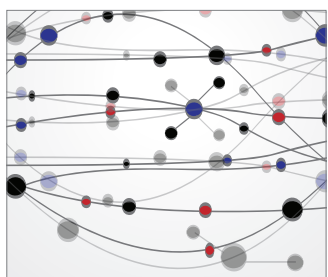

The Scientific World Journal
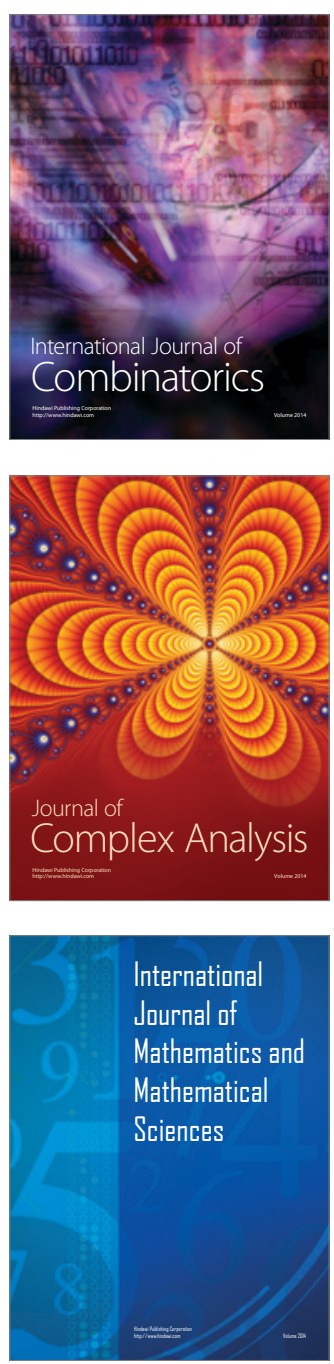
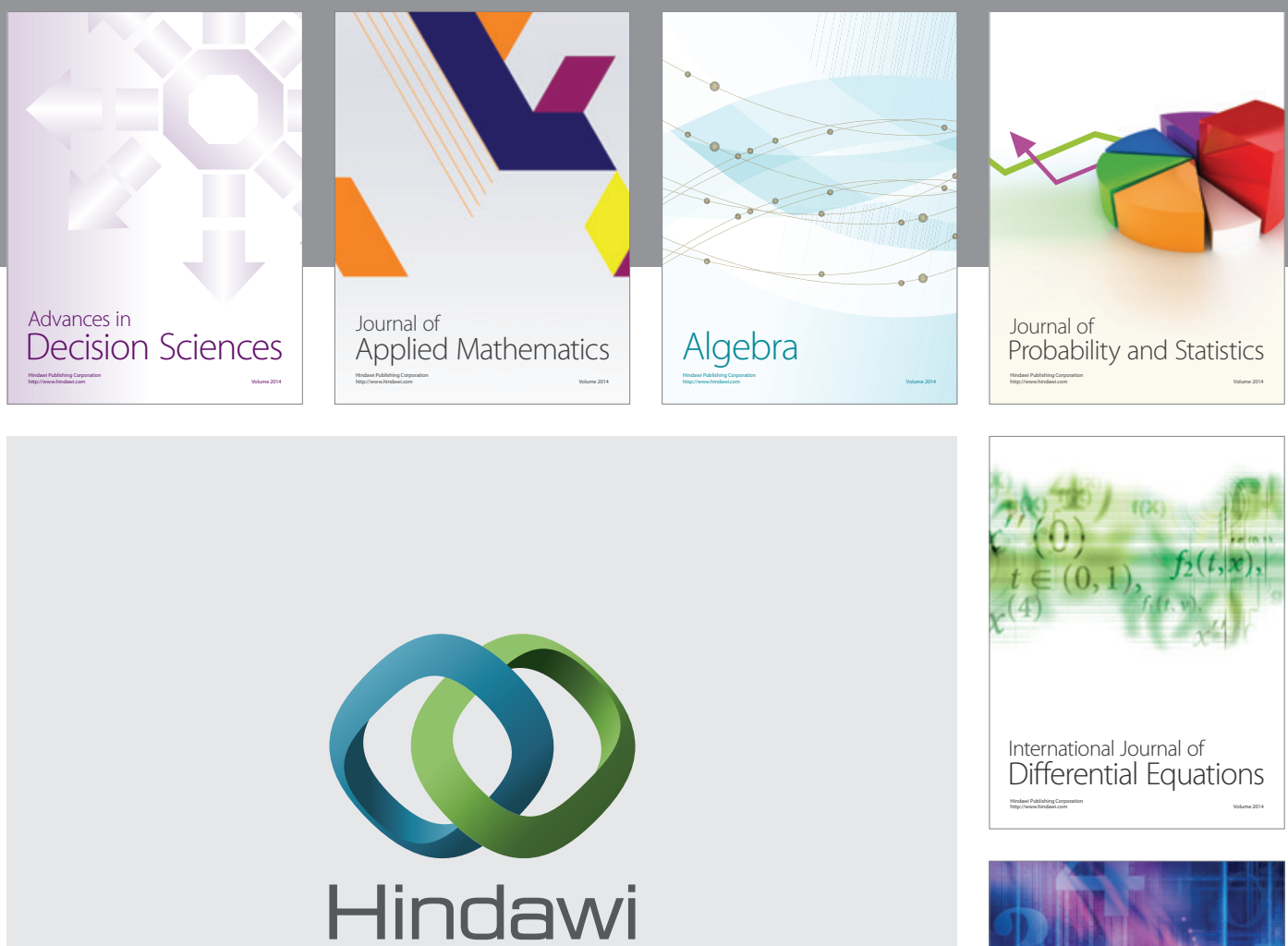

Submit your manuscripts at http://www.hindawi.com
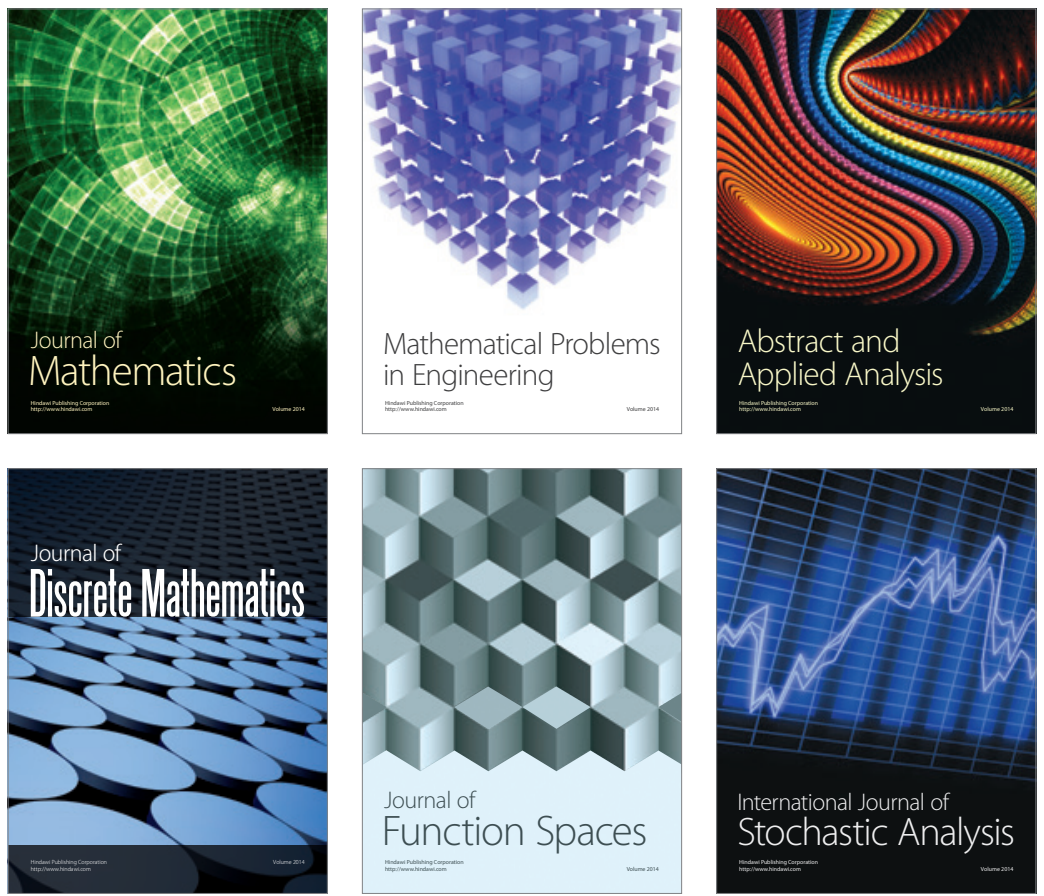

Journal of

Function Spaces

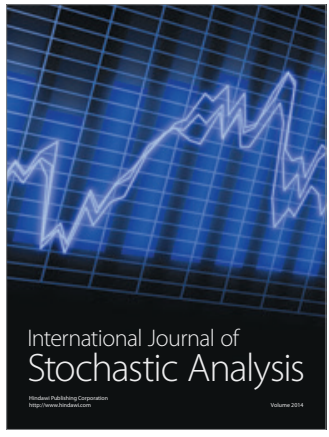

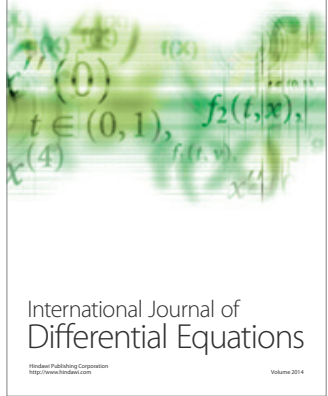
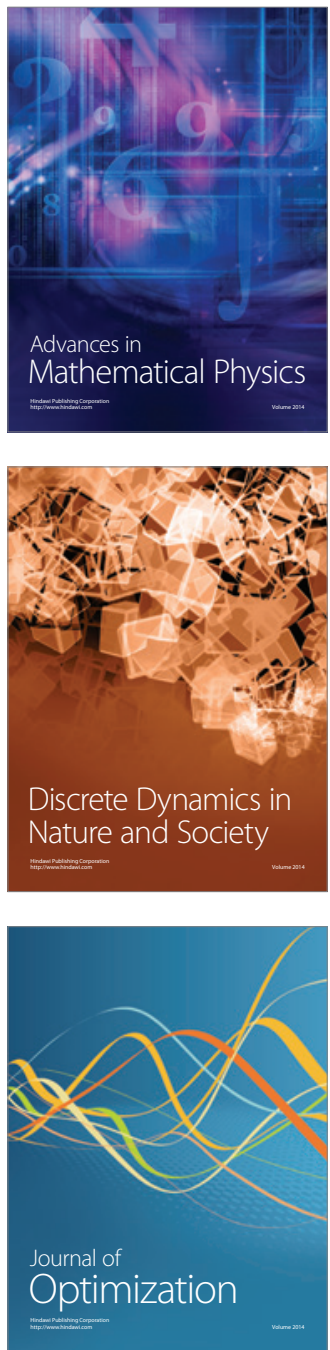